\title{
Preoperative Central Benzodiazepine Receptor Binding Potential and Cerebral Blood Flow Images on SPECT Predict Development of New Cerebral Ischemic Events and Cerebral Hyperperfusion After Carotid Endarterectomy
}

\author{
Yuiko Sato ${ }^{1,2}$, Kuniaki Ogasawara ${ }^{1,2}$, Hiroki Kuroda $^{1,2}$, Taro Suzuki ${ }^{1,2}$, Kohei Chida $^{1,2}$, Shunrou Fujiwara ${ }^{1}$, Kenta Aso $^{1,2}$, \\ Masakazu Kobayashi ${ }^{1,2}$, Kenji Yoshida ${ }^{1,2}$, Kazunori Terasaki ${ }^{1,2}$, and Akira Ogawa ${ }^{1,2}$ \\ ${ }^{1}$ Department of Neurosurgery, Iwate Medical University, Morioka, Japan; and ${ }^{2}$ Cyclotron Research Center, Iwate Medical University, \\ Morioka, Japan
}

Risk factors for cerebrovascular complications developing during or after carotid endarterectomy (CEA) include preoperative impairments in cerebral hemodynamics, as detected by the demonstration of decreased cerebrovascular reactivity (CVR) to acetazolamide on brain perfusion SPECT. Central benzodiazepine receptor binding potential (CBRBP) and cerebral blood flow (CBF) images on SPECT provide high sensitivity and high specificity for detecting misery perfusion in patients with chronic unilateral major cerebral artery occlusive disease. The aim of the present study was to determine whether preoperative CBRBP/CBF images on SPECT could identify patients at risk for new cerebral ischemic events, including neurologic deficits and cerebral ischemic lesions on diffusionweighted $\mathrm{MRI}$, or cerebral hyperperfusion after CEA and to compare the predictive accuracy of CBRBP/CBF with that of CVR to acetazolamide on SPECT. Methods: CBF, CVR, and CBRBP were assessed using $N$-isopropyl- $p$-123/-iodoamphetamine ( ${ }^{123}$ I-IMP) and ${ }^{123}$-iomazenil SPECT before CEA in 112 patients with unilateral internal carotid artery stenosis $(\geq 70 \%)$. CBF measurement using 123/-IMP SPECT was also performed immediately and $3 \mathrm{~d}$ after CEA. A region of interest was automatically placed in the middle cerebral artery territory in both cerebral hemispheres using a 3-dimensional stereotactic region-of-interest template. Diffusion-weighted MRI was performed within $3 \mathrm{~d}$ before and $24 \mathrm{~h}$ after surgery. Results: A preoperative increase in the affected side-to-contralateral side asymmetry on CBRBP/CBF value was the only significant independent predictor of postoperative new cerebral ischemic events (95\% confidence intervals [Cl], 1.145-1.608; $P=$ $0.0004)$ or post-CEA hyperperfusion (95\% Cl, 1.244-2.252; $P=0.0007)$. There was no difference in the ability to predict post-CEA hyperperfusion when comparing the area under the receiver-operating-characteristic curve of the affected side-tocontralateral side asymmetry on CBRBP/CBF and that of the CVR in the affected hemisphere. However, the former value $(0.924 ; 95 \% \mathrm{Cl}, 0.854-0.972)$ was significantly greater than the latter value $(0.782 ; 95 \% \mathrm{Cl}, 0.697-0.852)$ for the prediction

\footnotetext{
Received Jan. 13, 2011; revision accepted May 24, 2011.

For correspondence or reprints contact: Kuniaki Ogasawara, Department of Neurosurgery, Iwate Medical University, 19-1 Uchimaru, Morioka, 0208505 Japan.

E-mail: kuogasa@iwate-med.ac.jp

Published online Jul. 27, 2011.

COPYRIGHT @ 2011 by the Society of Nuclear Medicine, Inc.
}

of new postoperative cerebral ischemic events $(P<0.05)$. Conclusion: Preoperative CBRBP/CBF images on SPECT can more accurately identify patients at risk for cerebrovascular complications occurring during or after CEA when compared with preoperative CVR to acetazolamide.

Key Words: carotid endarterectomy; cerebral ischemic event; cerebral hyperperfusion; ${ }^{123}$-iomazenil; SPECT

J Nucl Med 2011; 52:1400-1407

DOI: 10.2967/jnumed.111.087940

$\mathbf{N}$ eurologic deficits after carotid endarterectomy (CEA) are rare, occurring in up to $5 \%$ of patients $(1-3)$. Hemodynamic cerebral ischemia due to hemispheric hypoperfusion during internal carotid artery (ICA) clamping and the generation of emboli from the surgical site play significant roles in the development of new cerebral ischemic events, including new neurologic deficits and new cerebral ischemic lesions on diffusion-weighted MRI (DWI) after CEA (4-11). Cerebral hyperperfusion syndrome is also a complication after CEA and is characterized by unilateral headache, face and eye pain, seizure, and focal symptoms that occur secondary to cerebral edema or intracerebral hemorrhage (12-15). In addition, recent studies have demonstrated that post-CEA hyperperfusion, even when asymptomatic, causes postoperative cortical neural damage that results in postoperative cognitive impairment (16-18).

Risk factors for the development of cerebrovascular complications associated with CEA include preoperative impairments in cerebral hemodynamics (19-24). Several investigators have demonstrated that a decrease in cerebrovascular reactivity (CVR) to acetazolamide, as measured using SPECT, predicts postoperative new cerebral ischemic events due to hemispheric hypoperfusion during ICA clamping (19) or emboli from the surgical site (20), or post-CEA hyperperfusion (22-24). However, acetazolamide is associated with frequent and various adverse side effects, including metabolic acidosis, hypokalemia, numbness of the 
extremities, headache, tinnitus, gastrointestinal disturbances, and Stevens-Johnson syndrome $(25,26)$. In fact, 1 study demonstrated that $63 \%$ of patients who underwent a SPECT study with acetazolamide challenge developed adverse effects between 1 and $3 \mathrm{~h}$ after administration of acetazolamide, and these symptoms lasted for 0.5-72 h (27) and frequently affected patients' activities of daily living, including the ability to engage in their jobs (27). Thus, it would be beneficial to develop a different SPECT method of predicting the cerebrovascular complications associated with CEA that does not require administration of acetazolamide.

The distribution of central benzodiazepine receptors in the cerebral cortex of the human brain has been widely studied with SPECT using ${ }^{123}$ I-iomazenil ( $\left.{ }^{123} \mathrm{I}-\mathrm{IMZ}\right)$ (2831). Central benzodiazepine receptor binding potential (CBRBP) on ${ }^{123}$ I-IMZ SPECT images is associated with neural density in the cerebral cortex, and a reduction in cortical CBRBP indicates cortical neural damage or loss (29-31). In the cerebral cortex with reduced CBRBP, cerebral metabolic rate of oxygen $\left(\mathrm{CMRO}_{2}\right)$ probably decreases in proportion to the degree of neuronal damage. In fact, CBRBP images on ${ }^{123}$ I-IMZ SPECT reportedly correlate with $\mathrm{CMRO}_{2}$ images on PET in the cerebral cortex in patients with carotid artery occlusive diseases $(32,33)$. Because oxygen extraction fraction (OEF), one of the key parameters of cerebral hemodynamics $(34,35)$, is a function of $\mathrm{CMRO}_{2}$ and cerebral blood flow (CBF), $\mathrm{CBRBP} / \mathrm{CBF}$ may reflect OEF. Indeed, a recent study has demonstrated that CBRBP/CBF images on SPECT correlate with $\mathrm{OEF}$ images on $\mathrm{PET}$ and provide high sensitivity and high specificity for detecting misery perfusion in the cerebral cortex in patients with chronic unilateral major cerebral artery occlusive disease (33).

Therefore, the aim of the present study was to determine whether preoperative CBRBP/CBF images on SPECT could identify patients at risk for new cerebral ischemic events or cerebral hyperperfusion after CEA and to compare the predictive accuracy of CBRBP/CBF with that of CVR to acetazolamide on SPECT.

\section{MATERIALS AND METHODS}

\section{Patients}

One hundred twelve patients (105 men and 7 women), aged 44 82 y (mean age, $69 \mathrm{y}$ ), with unilateral ICA stenosis $(\geq 70 \%)$ and useful residual function (modified Rankin disability scale 0 , 1 , or 2) who underwent CEA were enrolled in the present study. Concomitant disease states and symptoms were recorded, including 102 patients with hypertension, 42 patients with diabetes mellitus, and 61 patients with hyperlipidemia. Although 75 patients showed ischemic symptoms in the ipsilateral carotid territory, 37 patients exhibited asymptomatic ICA stenosis.

All patients underwent preoperative angiography with arterial catheterization. The overall average degree of ICA stenosis was $87.5 \% \pm 8.3 \%$, with a range of $70 \%-99 \%$, as per the North American Symptomatic Carotid Endarterectomy Trial (3). No patient had occlusion or stenosis of greater than $50 \%$ in the contralateral ICA or middle cerebral artery (MCA).
This protocol was reviewed and approved by the institutional ethics committee, and written informed consent was obtained from all patients or their next of kin.

\section{Brain SPECT Study}

Brain SPECT studies were performed using a ring-type SPECT scanner (Headtome-SET080; Shimadzu Corp.) (33,36), which provided 31 tomographic images simultaneously. The spatial resolution of the scanner with a low-energy, all-purpose collimator was $13 \mathrm{~mm}$ in full width at half maximum at the center of the field of view, and the slice thickness was $25 \mathrm{~mm}$ in full width at half maximum at the center of the field of view. Image slices were taken at 5-mm center-to-center spacing, parallel to the orbitomeatal line. The images were reconstructed using the weighted-filtered backprojection technique, in which the attenuation correction was made by detecting the edge of the object. An attenuation coefficient of $0.065 \mathrm{~cm}^{-1}$, a Butterworth filter (cutoff, $0.45 \mathrm{cycle} / \mathrm{cm}$; order, 3), and a ramp filter were used for image reconstruction.

CBF was assessed using $N$-isopropyl- $p$ - ${ }^{123}$ I-iodoamphetamine (123I-IMP) and SPECT 7-10 d before and immediately after CEA. The ${ }^{123}$ I-IMP SPECT study with and without acetazolamide challenge was performed as described previously (36). Preoperatively, $3 \mathrm{~d}$ after measurement of CBF without acetazolamide challenge, subjects underwent SPECT with acetazolamide challenge. In addition, patients with post-CEA hyperperfusion underwent a third $\mathrm{CBF}$ measurement in the same manner $3 \mathrm{~d}$ after CEA. The CBF images were calculated according to the ${ }^{123}$ I-IMP autoradiography method $(36,37)$.

The distribution of CBRBP in the cerebral cortex was assessed using ${ }^{123}$ I-IMZ SPECT between 2 and $7 \mathrm{~d}$ after ${ }^{123}$ I-IMP SPECT study with acetazolamide challenge. Subjects received approximately $167 \mathrm{MBq}$ of ${ }^{123} \mathrm{I}-\mathrm{IMZ}$ by intravenous bolus injection of 1.5 $\mathrm{mL}$ of solution into the cubital vein. One hundred eighty minutes later, scans were initiated, with a scanning duration of 23 min (33).

All SPECT images were transformed into the standard brain size and shape by linear and nonlinear transformation using SPM99 for anatomic standardization (38). Thus, brain images from all subjects had the same anatomic format. For each pixel in SPECT images obtained before surgery, 2 values were calculated as follows: CBRBP/CBF (count $/ \mathrm{mL} / 100 \mathrm{~g}$ brain $/ \mathrm{min})=$ radioactive counts of ${ }^{123} \mathrm{I}$-IMZ/CBF without acetazolamide challenge and $\operatorname{CVR}(\%)=$ ([CBF with acetazolamide challenge $-\mathrm{CBF}$ without acetazolamide challenge $] / \mathrm{CBF}$ without acetazolamide challenge) $\times 100$. Then, in all CBF, CBRBP/CBF, and CVR SPECT images, 318 constant regions of interest (ROIs) were automatically placed in both the cerebral and cerebellar hemispheres using a 3-dimensional stereotactic ROI template (39). The ROIs were grouped into 10 segments (callosomarginal, pericallosal, precentral, central, parietal, angular, temporal, posterior, hippocampus, and cerebellum) in each hemisphere according to the arterial supply. Five (precentral, central, parietal, angular, and temporal) of these 10 segments were combined and defined as an ROI perfused by the MCA (Fig. 1). The mean value of all pixels in the MCA ROI in each hemisphere was calculated.

Further, in preoperative CBRBP/CBF images of each patient, the asymmetry index (AI) was calculated as follows: CBRBP/CBF $\mathrm{AI}=\left(\mathrm{CBRBP} / \mathrm{CBF}_{\mathrm{CEA}}\right) /\left(\mathrm{CBRBP} / \mathrm{CBF}_{\mathrm{CON}}\right) \times 100$, where $\mathrm{CBRBP} / \mathrm{CBF}_{\mathrm{CEA}}$ is the value of the MCA ROI in the cerebral hemisphere ipsilateral to $\mathrm{CEA}$, and $\mathrm{CBRBP} / \mathrm{CBF}_{\mathrm{CON}}$ is that in the contralateral cerebral hemisphere. Using the same method, we studied 10 healthy subjects ( 8 men and 2 women; age, 35- 


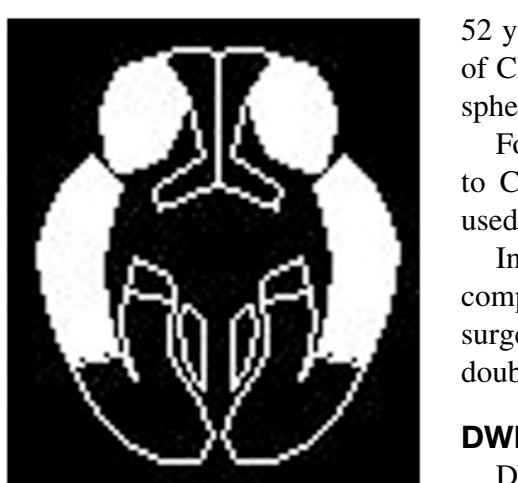

$52 \mathrm{y}$; mean age, 39 y) to obtain control values. The control value of CBRBP/CBF AI was $100.1 \pm 5.0$ when the left cerebral hemisphere was defined as the affected side (33).

For CVR in the MCA ROI in the cerebral hemisphere ipsilateral to CEA, data described previously $(36.8 \% \pm 9.2 \%)(20)$ were used as control values.

In $\mathrm{CBF}$ images of each patient, post-CEA hyperperfusion, compared with preoperative values in an MCA ROI ipsilateral to surgery, was defined as a CBF increase of $100 \%$ or more (i.e., a doubling) (23).

\section{DWI}

DWI was performed using a 1.5 -T whole-body imaging system (Signa MR/I; GE Healthcare) within $3 \mathrm{~d}$ before and $24 \mathrm{~h}$ after surgery. The images were acquired using single-shot spin-echo echo-planar imaging.

A neuroradiologist who was unaware of the patients' clinical information was used to analyze the images and determine development of new postoperative ischemic lesions.

\section{Assessment of Neurologic Deficits}

All patients were neurologically tested immediately before induction of general anesthesia and after recovery from general anesthesia by a neurologist who was unaware of the patients' clinical information, and the presence or absence of new postoperative neurologic deficits was recorded.

\section{Intraoperative and Postoperative Management}

All patients received antiplatelet therapy until the morning of the day on which CEA was performed. Further, all patients underwent surgery under general anesthesia. Blood pressure was kept stable in a range $\pm 20 \%$ of the preoperative level throughout the procedure by adjusting the depth of anesthesia or, if needed, by intravenous administration of a vasodilator (nitroglycerin) or a vasoconstrictor (theoadrenalin). An intraluminal shunt was not used in these procedures. The mean duration of ICA clamping was $36 \mathrm{~min}$, ranging from 21 to $48 \mathrm{~min}$. In all patients with post-CEA hyperperfusion, intensive control of arterial blood pressure between 100 and $140 \mathrm{~mm} \mathrm{Hg}$ was instituted using intravenous administration of antihypertensive drugs immediately after SPECT. When CBF decreased and hyperperfusion resolved on the third postoperative day, pharmacologic control of blood pressure was discontinued. However, when hyperperfusion persisted, systolic arterial blood pressure was maintained below $140 \mathrm{~mm} \mathrm{Hg}$. When hyperperfusion syndrome developed, the patient was placed in propofol coma. A diagnosis of hyperperfusion syndrome required seizure, alteration in consciousness level, or focal neurologic signs such as motor weakness that developed or worsened between $24 \mathrm{~h}$ and $14 \mathrm{~d}$ after surgery, and hyperperfusion on the ${ }^{123}$ I-IMP SPECT performed after CEA.

\section{Statistical Analysis}

Data are expressed as the mean \pm SD. The relationship between each variable and the development of new postoperative cerebral ischemic events (neurologic deficits or cerebral ischemic lesions on DWI) or post-CEA hyperperfusion was evaluated with univariate analysis using the Mann-Whitney $U$ test or $\chi^{2}$ test. A multivariate statistical analysis of factors related to the development of new postoperative cerebral ischemic events or post-CEA hyperperfusion was also performed using a logistic regression model. Variables with a $P$ value of less than 0.2 in the univariate analyses were selected for analysis in the final model. However, 
preoperative CVR in the affected hemisphere was not adopted in the model because previous studies have already established the strong relationship between this factor and the development of new postoperative cerebral ischemic events or post-CEA hyperperfusion $(19,20,22-24)$. Differences were deemed statistically significant if the $P$ value was less than 0.05 . To estimate the ability to discriminate between patients with and without new postoperative cerebral ischemic events or post-CEA hyperperfusion, a receiver-operating-characteristic (ROC) curve was constructed, plotting sensitivity versus 1 minus specificity for possible cutoff values, and the area under the ROC curve was calculated. The ROC curve was calculated in increments or decrements of 0.5 $\mathrm{SD}$ (2.5 for CBRBP/CBF AI; $4.6 \%$ for CVR) from the mean value (100.1 for CBRBP/CBF AI; $36.8 \%$ for CVR) obtained in healthy subjects. Differences in the ROC area between the CBRBP/CBF $\mathrm{AI}$ and CVR in the affected hemisphere were analyzed using $95 \%$ confidence intervals (CIs).

\section{RESULTS}

\section{Incidence and Course of Cerebrovascular Complications After CEA}

Of the 112 patients studied, 9 patients (8\%) developed new postoperative ischemic lesions on DWI in the cerebral hemisphere ipsilateral to CEA. All the new ischemic lesions were spotty and their diameters were $1.5 \mathrm{~cm}$ or less. Four patients (4\%) developed new postoperative neurologic deficits: $2(2 \%)$ with new postoperative cerebral ischemic lesions on DWI and $2(2 \%)$ without. Although those deficits resolved completely within $12 \mathrm{~h}$ in 3 patients, the neurologic deficits in the remaining patient persisted for more than $24 \mathrm{~h}$ after surgery. Thus, a total of 11 patients (10\%) experienced new postoperative ischemic events (postoperative cerebral ischemic lesions on DWI or postoperative neurologic deficits).

Thirteen patients $(12 \%)$ met CBF criteria for post-CEA hyperperfusion on the CBF SPECT images obtained immediately after surgery. Of these 13 patients, 2 exhibited new transient neurologic deficits on recovery from general anesthesia after surgery with (Fig. 2) or without new postoperative cerebral ischemic lesions on DWI, respectively.

In 11 of the 13 patients with hyperperfusion immediately after CEA, hyperperfusion was not present on the SPECT study performed on the third postoperative day, and these 11 patients had uneventful postoperative courses. However, the remaining 2 patients with cerebral hyperperfusion immediately after CEA experienced a progressive increase in CBF on the third postoperative day. Of these 2 patients, 1 who exhibited right transient hemiparesis on recovery from general anesthesia after surgery and who exhibited new postoperative cerebral ischemic lesions on DWI developed hyperperfusion syndrome with confusion and aphasia on the fourth postoperative day (Fig. 2). Another patient experienced cerebral hyperperfusion syndrome with confusion and right motor weakness with onset on the sixth postoperative day. Propofol coma was induced in both patients. After termination of the propofol coma, both patients eventually experienced full recovery.

\section{Factors Related to New Postoperative Ischemic Events}

Results of univariate analysis of factors related to the development of new postoperative ischemic events are summarized in Table 1. The preoperative CBRBP/CBF AI was significantly higher and the CVR in the affected hemisphere was significantly lower in patients with new postoperative ischemic events than in those without. Other variables were not significantly associated with the development of new postoperative ischemic events. After eliminating closely related variables in the univariate analyses, the following confounders $(P<0.2)$ were adopted in the logistic regression model for the multivariate analysis: sex, degree of ICA stenosis, and preoperative $\mathrm{CBRBP} / \mathrm{CBF} \mathrm{AI}$. The multivariate analysis revealed that high preoperative $\mathrm{CBRBP} / \mathrm{CBF} \mathrm{AI}$ was significantly associated with the development of new postoperative ischemic events $(95 \% \mathrm{CI}, 1.145-1.608 ; P=$ $0.0004)$.
A

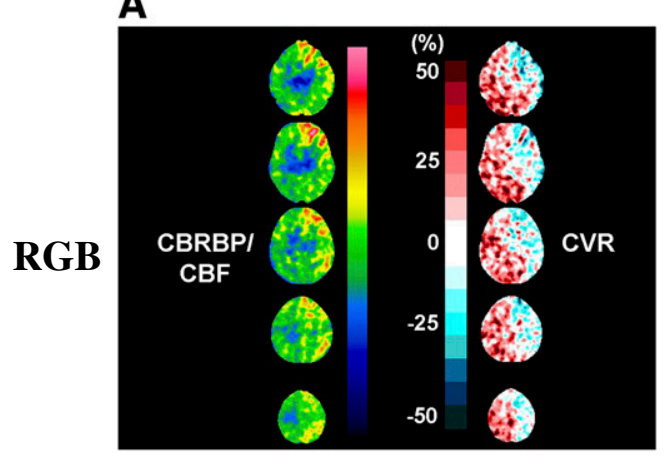

B

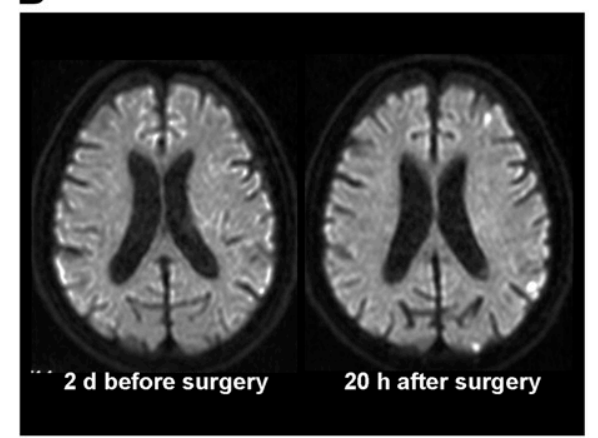

C

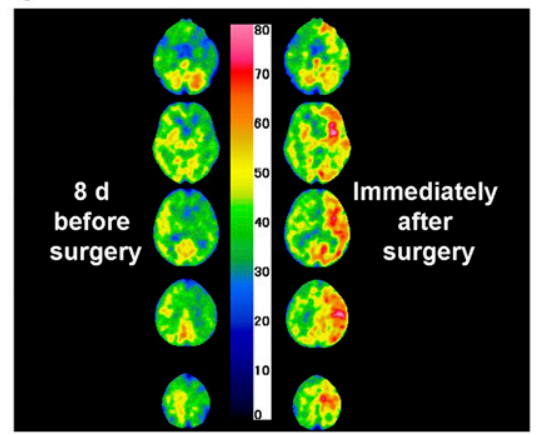

FIGURE 2. A 64-y-old man with symptomatic left internal carotid artery stenosis (95\%) exhibiting slight right hemiparesis on recovery from general anesthesia after CEA. Six hours later, hemiparesis resolved. However, hyperperfusion syndrome with confusion and aphasia developed on fourth postoperative day. (A) Preoperative CBRBP/CBF and CVR to acetazolamide SPECT images show elevation of values in left cerebral cortex, compared with those in right cerebral cortex, and reduction of values in cerebral hemisphere, respectively. (B) Diffusion-weighted MR image before surgery shows no abnormal findings in left cerebral hemisphere, in which new multiple high intense lesions developed after surgery. (C) CBF SPECT images before surgery show hypoperfusion in left cerebral hemisphere, in which hyperperfusion developed immediately after surgery. 
TABLE 1

Risk Factors for Development of New Postoperative Cerebral Ischemic Events

\begin{tabular}{|c|c|c|c|}
\hline \multirow[b]{2}{*}{ Risk factor } & \multicolumn{2}{|c|}{ New postoperative cerebral ischemic events } & \multirow[b]{2}{*}{$P$} \\
\hline & Yes $(n=11)$ & No $(n=101)$ & \\
\hline Age (y) & $71.2 \pm 5.3$ & $68.4 \pm 6.8$ & 0.2767 \\
\hline Male sex & $9(82)$ & $96(95)$ & 0.1404 \\
\hline Hypertension & $10(91)$ & $92(91)$ & $>0.9999$ \\
\hline Diabetes mellitus & $3(27)$ & 39 (39) & 0.5318 \\
\hline Hyperlipidemia & $6(55)$ & $55(54)$ & $>0.9999$ \\
\hline Symptomatic lesions & $9(82)$ & $66(65)$ & 0.3332 \\
\hline Degree of ICA stenosis (\%) & $89.5 \pm 8.8$ & $87.2 \pm 8.3$ & 0.1337 \\
\hline Duration of ICA clamping (min) & $35.5 \pm 6.4$ & $36.2 \pm 5.5$ & 0.6915 \\
\hline Preoperative CBRBP/CBF Al & $110.8 \pm 6.6$ & $102.1 \pm 5.0$ & $<0.0001$ \\
\hline Preoperative CVR to acetazolamide (\%) & $13.6 \pm 14.1$ & $28.7 \pm 11.8$ & 0.0010 \\
\hline
\end{tabular}

Figure 3A illustrates the ROC curves of the CBRBP/CBF $\mathrm{AI}$ and the CVR to acetazolamide in the affected hemisphere, which can be taken as a measure of their ability to predict the development of new postoperative ischemic events. The ROC area of the CBRBP/CBF AI $(0.924 ; 95 \%$ CI, 0.854-0.972) was significantly greater than that of the CVR (0.782; 95\% CI, 0.697-0.852) $(P<0.05)$.

Sensitivity and specificity for the CBRBP/CBF AI in the cutoff point lying closest to the left upper corner of the ROC curve in predicting development of new postoperative ischemic events were $91 \%(10 / 11)$ and $73 \%(74 / 101)$ (cutoff point, 105.1: the mean $+\mathrm{SD}$ of the control value obtained from healthy subjects), respectively (Figs. 3A and 4). In the cutoff point, positive and negative predictive values were 27\% (10/37) and 99\% (74/75), respectively.

Subgroup analysis of patients with and without development of new neurologic deficits immediately after surgery, regardless of whether they developed new postoperative cerebral ischemic lesions on DWI, was performed in the same manner. Results of univariate analysis of factors related to the development of new postoperative neurologic deficits are summarized in Supplemental Table 1 (supplemental materials are available online only at http://jnm. snmjournals.org). The preoperative CBRBP/CBF AI was

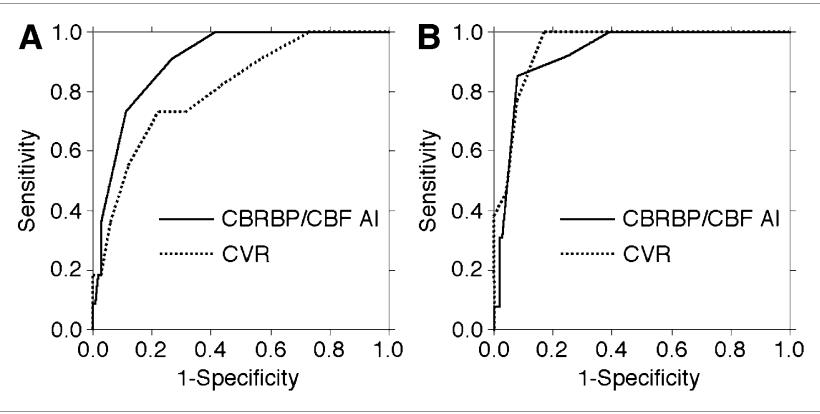

FIGURE 3. ROC curves used to determine accuracy of CBRBP/ CBF Al and CVR to acetazolamide in prediction of new postoperative ischemic events (A) and postoperative hyperperfusion (B). significantly higher in patients with new postoperative neurologic deficits than in those without. Other variables, including CVR to acetazolamide, were not significantly associated with the development of new postoperative neurologic deficits. After eliminating closely related variables in the univariate analyses, the following confounders $(P<$ 0.2 ) were adopted in the logistic regression model for the multivariate analysis: degree of ICA stenosis and preoperative CBRBP/CBF AI. The multivariate analysis revealed that high preoperative CBRBP/CBF AI was significantly associated with the development of new postoperative neurologic deficits (95\% CI, 1.002-1.156; $P=0.0477$ ).

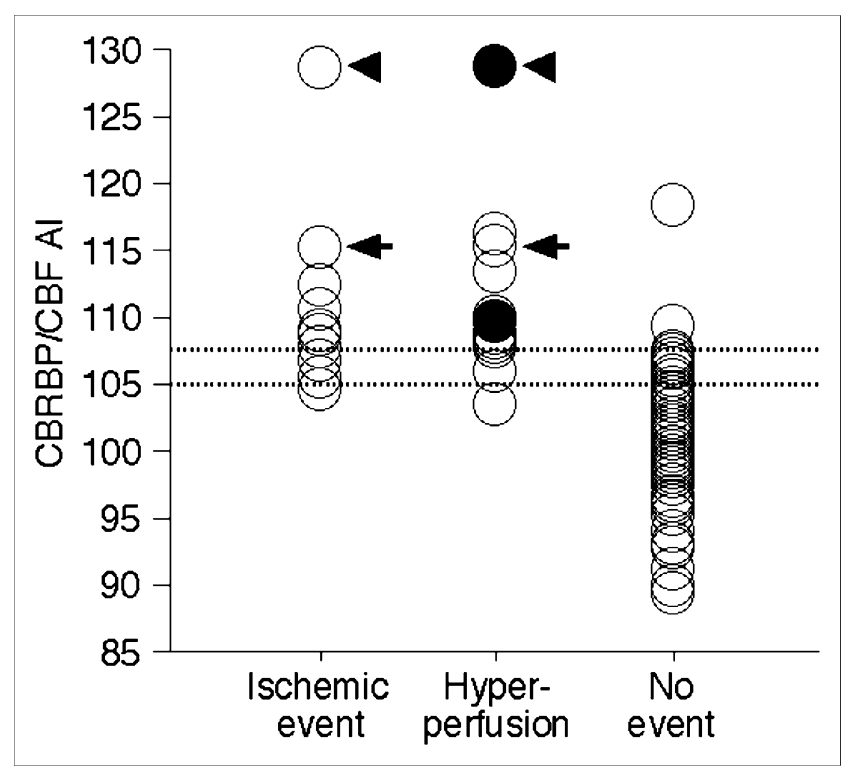

FIGURE 4. Relationship between CBRBP/CBF Al, postoperative ischemic events, and postoperative hyperperfusion. Arrows or arrowheads indicate identical patient with both postoperative ischemic events and postoperative hyperperfusion. Closed circles indicate patients with cerebral hyperperfusion syndrome. Each dashed horizontal line denotes mean + 1.5 (107.6) or mean + 1 (105.1) SD of CBRBP/CBF Al obtained in healthy volunteers, respectively. 
Supplemental Figure 1 shows ROC curves of the CBRBP/CBF AI and the CVR to acetazolamide in the affected hemisphere, which can be taken as a measure of their ability to predict development of new postoperative neurologic deficits. The ROC area of the CBRBP/CBF AI (0.892; 95\% CI, 0.815-0.941) was significantly greater than that of the CVR $(0.737$; 95\% CI, 0.647-0.813) $(P<0.05)$.

Sensitivity and specificity for the CBRBP/CBF AI in the cutoff point lying closest to the left upper corner of the ROC curve in predicting development of new postoperative neurologic deficits were 100\% (4/4) and 69\% (75/108) (cutoff point, 105.1: the mean + SD of the control value obtained from healthy subjects), respectively (Supplemental Fig. 1). In the cutoff point, positive and negative predictive values were $11 \%(4 / 37)$ and 100\% (75/75), respectively.

\section{Factors Related to Post-CEA Hyperperfusion}

Results of univariate analysis of factors related to the development of cerebral hyperperfusion after CEA are summarized in Table 2. The preoperative CBRBP/CBF AI was significantly higher and the CVR in the affected hemisphere was significantly lower in patients with post-CEA hyperperfusion than in those without. Other variables were not significantly associated with the development of postCEA hyperperfusion. After closely related variables in the univariate analyses were eliminated, the following confounders $(P<0.2)$ were adopted in the logistic regression model for the multivariate analysis: symptomatic lesions, degree of ICA stenosis, and preoperative CBRBP/CBF AI. The multivariate analysis revealed that high preoperative CBRBP/CBF AI was significantly associated with the development of postoperative cerebral hyperperfusion (95\% CI, 1.244-2.252; $P=0.0007$ ).

Figure $3 \mathrm{~B}$ illustrates ROC curves of the CBRBP/CBF AI and the CVR in the affected hemisphere, which can be taken as a measure of their ability to predict development of post-CEA hyperperfusion. There was no significant difference when comparing the ROC area of the CBRBP/CBF AI $(0.925 ; 95 \% \mathrm{CI}, 0.860-0.966)$ and that of the CVR (0.954; 95\% CI, 0.897-0.985).

Sensitivity and specificity for the CBRBP/CBF AI in the cutoff point lying closest to the left upper corner of the ROC curve in predicting development of post-CEA hyperperfusion were $85 \%(11 / 13)$ and $92 \%$ (91/99) (cutoff point, 107.6: the mean +1.5 SDs of the control value obtained from healthy subjects), respectively (Figs. 3B and 4). In the cutoff point, positive and negative predictive values were $58 \%(11 / 19)$ and $98 \%$ (91/93), respectively.

\section{DISCUSSION}

The present study demonstrated that preoperative CBRBP/ CBF images on SPECT can more accurately identify patients at risk for cerebrovascular complications occurring during or after CEA when compared with preoperative CVR to acetazolamide.

Hemodynamic compromise due to chronic cerebral ischemia in ICA stenosis implies an inadequate collateral blood flow to maintain the $\mathrm{CBF}$ against a further decrease in perfusion pressure. Because ICA clamping during CEA may cause a critical reduction of cerebral perfusion in the area with the hemodynamic compromise, cerebral ischemic events may develop more frequently in patients with hemodynamic compromise than in those without. One study reported that reduction of preoperative cerebrovascular reactivity to acetazolamide measured by brain perfusion SPECT correlates with a high risk of significant cerebral ischemia during ICA clamping (19).

Microembolic signals on intraoperative transcranial Doppler monitoring of the MCA are detected in more than $90 \%$ of patients undergoing CEA $(4,5)$. In particular, there is a significant correlation between the number of microembolic signals during dissection of the carotid arteries and development of new postoperative ischemic lesions on DWI (4-9). According to the concept proposed by Caplan and Hennerici (40), preexisting hemodynamic impairment may facilitate the onset of ischemia due to emboli generated from a proximal lesion. One study has demonstrated that reduction of preoperative cerebrovascular reactivity to acetazolamide measured by brain perfusion SPECT predicts the development of new postoperative cerebral ischemic lesions on DWI that are caused by microemboli generated during carotid dissection in CEA (20).

Mechanisms for the development of post-CEA hyperperfusion have been proposed as follows (14): severely reduced cerebral perfusion pressure due to severe ICA stenosis and deficient collateral circulation may lead to maximal dilation of resistance vessels by cerebrovascular autoregulation; after restoration of normal perfusion pressure after CEA, chronically impaired autoregulatory mechanisms may require several days to adjust to the new steady state, resulting in hyperperfusion in the interim. Several investigators have demonstrated that reduction of preoperative cerebrovascular reactivity to acetazolamide measured by brain perfusion SPECT predicts the development of cerebral hyperperfusion after CEA (21-24).

The present study demonstrated that high preoperative CBRBP/CBF AI was a significant independent predictor of both postoperative new cerebral ischemic events and postCEA hyperperfusion. Because high CBRBP/CBF AI suggests the presence of misery perfusion in the affected hemisphere (33) and resistance vessels maximally dilate due to autoregulatory mechanisms in the misery perfusion, our findings support the theory that preoperative hemodynamic impairment is associated with development of cerebral ischemic events due to intraoperative emboli as well as significant hemispheric hypoperfusion during ICA clamping and that cerebral hyperperfusion results from loss of normal vasoconstriction secondary to chronic cerebral ischemia and maladaptive autoregulatory mechanisms.

The ROC analysis in the present study showed that although the ability to predict development of post-CEA hyperperfusion was similar when comparing CBRBP/CBF AI and preoperative CVR to acetazolamide, the former value 
TABLE 2

Risk Factors for Development of Postoperative Cerebral Hyperperfusion on SPECT

\begin{tabular}{|c|c|c|c|}
\hline \multirow[b]{2}{*}{ Risk factor } & \multicolumn{2}{|c|}{ Postoperative hyperperfusion } & \multirow[b]{2}{*}{$P$} \\
\hline & Yes $(n=13)$ & No $(n=99)$ & \\
\hline Age (y) & $70.8 \pm 6.3$ & $68.4 \pm 6.8$ & 0.2726 \\
\hline Male sex & $13(100)$ & $92(93)$ & $>0.9999$ \\
\hline Hypertension & $13(100)$ & $89(90)$ & 0.6024 \\
\hline Diabetes mellitus & $3(23)$ & 39 (39) & 0.3643 \\
\hline Hyperlipidemia & $9(69)$ & $52(53)$ & 0.3759 \\
\hline Symptomatic lesions & $12(92)$ & $63(64)$ & 0.0570 \\
\hline Degree of ICA stenosis (\%) & $89.5 \pm 9.8$ & $87.2 \pm 8.1$ & 0.0785 \\
\hline Duration of ICA clamping (min) & $35.3 \pm 4.0$ & $36.3 \pm 5.6$ & 0.6198 \\
\hline Preoperative CBRBP/CBF AI & $111.2 \pm 6.3$ & $101.9 \pm 4.8$ & $<0.0001$ \\
\hline Preoperative CVR to acetazolamide (\%) & $6.3 \pm 9.9$ & $29.9 \pm 10.5$ & $<0.0001$ \\
\hline
\end{tabular}

predicted development of new postoperative cerebral ischemic events more accurately than did the latter value. The CBRBP/ CBF AI also more accurately predicted development of new neurologic deficits immediately after surgery, regardless of whether there were new postoperative cerebral ischemic lesions on DWI. Severe preoperative impairment in cerebral hemodynamics is associated with even worse cerebral hemispheric hypoperfusion during ICA clamping or in the context of cerebral ischemia due to emboli generated from the surgical site $(19,20)$. In the physiologic process known as cerebrovascular autoregulation, misery perfusion is a state of severe hemodynamic impairment (35). Although there is a significant negative correlation between OEF and CVR to acetazolamide, the decrease in CVR and the increase in OEF do not always parallel each other: less than $50 \%$ of patients with reduced CVR have an elevated OEF corresponding with misery perfusion (41). In contrast, CBRBP/CBF AI on SPECT provides high sensitivity and high specificity for detection of elevated $\mathrm{OEF}$ in the cerebral cortex in patients with chronic unilateral major cerebral artery occlusive disease (33). The difference in the accuracy between CBRBP/CBF AI and CVR for the detection of misery perfusion may also explain the difference between these 2 parameters in their ability to predict new postoperative cerebral ischemic events. Thus, CBRBP/CBF AI may be a useful and alternative SPECT method of predicting the cerebrovascular complications associated with CEA that does not require administration of acetazolamide (with its frequent and various adverse side effects).

The present study possesses several limitations that require discussion. First, the present study included only patients with unilateral ICA stenosis and used affected side-to-contralateral side asymmetry on CBRBP/CBF SPECT images to detect hemodynamic impairment in the affected cerebral hemisphere. However, impairments in cerebral hemodynamics are more severe in patients with bilateral ICA stenoocclusive disease than in those with unilateral ICA stenosis (42), and impairments in bilateral cerebral hemodynamics in patients with bilateral major cerebral arterial occlusive disease may not be detected by the present SPECT method using affected side-to-contralateral side asymmetry. Although absolute quantification of CBRBP using SPECT has been reported (28), this issue of whether CBRBP/CBF values quantified by this method in patients with bilateral ICA stenosis predict development of the cerebrovascular complications associated with CEA remains unclear. Second, normal values were obtained from healthy subjects who were younger than patients with occlusive disease. However, affected-to-contralateral side asymmetry was used to determine CBRBP/ $\mathrm{CBF}$ value in the affected cerebral hemisphere, which may have minimized the effect of age difference between the 2 populations in the present study.

\section{CONCLUSION}

Preoperative CBRBP/CBF images on SPECT more accurately identify patients at risk for cerebrovascular complications occurring during or after CEA when compared with preoperative CVR to acetazolamide.

\section{DISCLOSURE STATEMENT}

The costs of publication of this article were defrayed in part by the payment of page charges. Therefore, and solely to indicate this fact, this article is hereby marked "advertisement" in accordance with 18 USC section 1734.

\section{ACKNOWLEDGMENTS}

No potential conflict of interest relevant to this article was reported.

\section{REFERENCES}

1. European Carotid Surgery Trialists' Collaborative Group. MRC European Carotid Surgery Trial: interim results for symptomatic patients with severe (70$99 \%$ ) or with mild (0-29\%) carotid stenosis. Lancet. 1991;337:1235-1243.

2. Executive Committee for the Asymptomatic Carotid Atherosclerosis Study. Endarterectomy for asymptomatic carotid artery stenosis. JAMA. 1995;273:1421-1428. 
3. North American Symptomatic Carotid Endarterectomy Trial Collaborators. Beneficial effect of carotid endarterectomy in symptomatic patients with high-grade carotid stenosis. N Engl J Med. 1991;325:445-453.

4. Wolf O, Heider P, Heinz M, et al. Microembolic signals detected by transcranial Doppler sonography during carotid endarterectomy and correlation with serial diffusion-weighted imaging. Stroke. 2004;35:e373-e375.

5. Ackerstaff RG, Moons KG, van de Vlasakker CJ, et al. Association of intraoperative transcranial doppler monitoring variables with stroke from carotid endarterectomy. Stroke. 2000;31:1817-1823.

6. Gavrilescu T, Babikian VL, Cantelmo NL, Rosales R, Pochay V, Winter M. Cerebral microembolism during carotid endarterectomy. Am J Surg. 1995;170:159-164.

7. Verhoeven BA, de Vries JP, Pasterkamp G, et al. Carotid atherosclerotic plaque characteristics are associated with microembolization during carotid endarterectomy and procedural outcome. Stroke. 2005;36:1735-1740.

8. Gaunt ME, Martin PJ, Smith JL, et al. Clinical relevance of intraoperative embolization detected by transcranial Doppler ultrasonography during carotid endarterectomy: a prospective study of 100 patients. Br J Surg. 1994;81:1435-1439.

9. Jansen C, Ramos LM, van Heesewijk JP, Moll FL, van Gijn J, Ackerstaff RG. Impact of microembolism and hemodynamic changes in the brain during carotid endarterectomy. Stroke. 1994;25:992-997.

10. Mille T, Tachimiri ME, Klersy C, et al. Near infrared spectroscopy monitoring during carotid endarterectomy: which threshold value is critical? Eur J Vasc Endovasc Surg. 2004;27:646-650.

11. van der Schaaf IC, Horn J, Moll FL, Ackerstaff RG. Transcranial Doppler monitoring after carotid endarterectomy. Ann Vasc Surg. 2005;19:19-24.

12. Piepgras DG, Morgan MK, Sundt TM Jr, Yanagihara T, Mussman LM. Intracerebral hemorrhage after carotid endarterectomy. J Neurosurg. 1988;68:532-536.

13. Sundt TM Jr, Sharbrough FW, Piepgras DG, Kearns TP, Messick JM Jr, O'Fallon WM. Correlation of cerebral blood flow and electroencephalographic changes during carotid endarterectomy, with results of surgery and hemodynamics of cerebral ischemia. Mayo Clin Proc. 1981;56:533-553.

14. Bernstein M, Fleming JF, Deck JH. Cerebral hyperperfusion after carotid endarterectomy: a cause of cerebral hemorrhage. Neurosurgery. 1984;15:50-56.

15. Solomon RA, Loftus CM, Quest DO, Correll JW. Incidence and etiology of intracerebral hemorrhage following carotid endarterectomy. J Neurosurg. 1986; 64:29-34.

16. Chida K, Ogasawara K, Suga Y, et al. Postoperative cortical neural loss associated with cerebral hyperperfusion and cognitive impairment following carotid endarterectomy: ${ }^{123}$ I-iomazenil SPECT study. Stroke. 2009;40:448-453.

17. Ogasawara K, Kobayashi M, Suga Y, et al. Significance of postoperative crossed cerebellar hypoperfusion in patients with cerebral hyperperfusion following carotid endarterectomy: SPECT study. Eur J Nucl Med Mol Imaging. 2008;35:146-152.

18. Ogasawara K, Yamadate K, Kobayashi M, et al. Postoperative cerebral hyperperfusion associated with impaired cognitive function in patients undergoing carotid endarterectomy. J Neurosurg. 2005;102:38-44.

19. Kim JS, Moon DH, Kim GE, et al. Acetazolamide stress brain-perfusion SPECT predicts the need for carotid shunting during carotid endarterectomy. J Nucl Med. 2000;41:1836-1841.

20. Aso K, Ogasawara K, Sasaki M, et al. Preoperative cerebrovascular reactivity to acetazolamide measured by brain perfusion SPECT predicts development of cerebral ischemic lesions caused by microemboli during carotid endarterectomy. Eur J Nucl Med Mol Imaging. 2009;36:294-301.

21. Fukuda T, Ogasawara K, Kobayashi M, et al. Prediction of cerebral hyperperfusion after carotid endarterectomy using cerebral blood volume measured by perfusion-weighted MR imaging compared with single-photon emission CT. AJNR Am J Neuroradiol. 2007;28:737-742.

22. Hosoda K, Kawaguchi T, Shibata Y, et al. Cerebral vasoreactivity and internal carotid artery flow help to identify patients at risk for hyperperfusion after carotid endarterectomy. Stroke. 2001;32:1567-1573.
23. Ogasawara K, Yukawa H, Kobayashi M, et al. Prediction and Monitoring of cerebral hyperperfusion after carotid endarterectomy by using single-photon emission computerized tomography scanning. J Neurosurg. 2003;99:504-510.

24. Yoshimoto T, Houkin K, Kuroda S, Abe H, Kashiwaba T. Low cerebral blood flow and perfusion reserve induce hyperperfusion after surgical revascularization: case reports and analysis of cerebral hemodynamics. Surg Neurol. 1997; 48:132-139.

25. Derick RJ. Carbonic anhydrase inhibitors. In: Mauger TF, Craig EL, eds. Hevener's Ocular Pharmacology. 6th ed. St Louis, MO: CV Mosby Co.; 1994:ch. 4.

26. Ogasawara K, Tomitsuka N, Kobayashi M, et al. Stevens-Johnson syndrome associated with intravenous acetazolamide administration for evaluation of cerebrovascular reactivity. Case report. Neurol Med Chir (Tokyo). 2006;46:161-163.

27. Saito H, Ogasawara K, Suzuki T, et al. Adverse effects of intravenous acetazolamide administration for evaluation of cerebrovascular reactivity using brain perfusion single-photon emission computed tomography in patients with major cerebral artery steno-occlusive diseases. Neurol Med Chir (Tokyo). In press.

28. Millet P, Graf C, Moulin M, Ibanez V. SPECT quantification of benzodiazepine receptor concentration using a dual-ligand approach. J Nucl Med. 2006;47:783792.

29. Nakagawara J, Sperling B, Lassen NA. Incomplete brain infarction of reperfused cortex may be quantitated with iomazenil. Stroke. 1997;28:124-132.

30. Hatazawa J, Satoh T, Shimosegawa E, et al. Evaluation of cerebral infarction with iodine 123-iomazenil SPECT. J Nucl Med. 1995;36:2154-2161.

31. Hatazawa J, Shimosegawa E, Satoh T, Kanno I, Uemura K. Central benzodiazepine receptor distribution after subcortical hemorrhage evaluated by means of $\left[{ }^{123}\right.$ I] iomazenil and SPECT. Stroke. 1995;26:2267-2271.

32. Dong Y, Fukuyama H, Nabatame H, Yamauchi H, Shibasaki H, Yonekura Y. Assessment of benzodiazepine receptors using iodine-123-labeled iomazenil singlephoton emission computed tomography in patients with ischemic cerebrovascular disease: a comparison with PET study. Stroke. 1997;28:1776-1782.

33. Chida K, Ogasawara K, Kuroda H, et al. Central benzodiazepine receptor binding potential and cerebral blood flow images on SPECT correlate with oxygen extraction fraction images on PET in cerebral cortex with unilateral major cerebral artery occlusive disease. J Nucl Med. 2011;52:511-518.

34. Gibbs JM, Wise RJ, Leenders KL, Jones T. Evaluation of cerebral perfusion reserve in patients with carotid-artery occlusion. Lancet. 1984;1:310-314.

35. Powers WJ. Cerebral hemodynamics in ischemic cerebrovascular disease. Ann Neurol. 1991;29:231-240.

36. Ogasawara K, Ito $\mathrm{H}$, Sasoh $\mathrm{M}$, et al. Quantitative measurement of regional cerebrovascular reactivity to acetazolamide using ${ }^{123} \mathrm{I}-\mathrm{N}$-isopropyl-p-iodoamphetamine autoradiography with SPECT: validation study using $\mathrm{H}_{2}{ }^{15} \mathrm{O}$ with PET. J Nucl Med. 2003;44:520-525.

37. Iida $\mathrm{H}$, Itoh $\mathrm{H}$, Nakazawa $\mathrm{M}$, et al. Quantitative mapping of regional cerebral blood flow using iodine-123-IMP and SPECT. J Nucl Med. 1994;35:2019-2030.

38. Friston KJ, Frith CD, Liddle PF, Dolan RJ, Lammertsma AA, Frackowiak RS. The relationship between global and local changes in PET scans. J Cereb Blood Flow Metab. 1990;10:458-466.

39. Takeuchi R, Matsuda H, Yoshioka K, Yonekura Y. Cerebral blood flow SPET in transient global amnesia with automated ROI analysis by 3DSRT. Eur J Nucl Med Mol Imaging. 2004;31:578-589.

40. Caplan LR, Hennerici M. Impaired clearance of emboli (washout) is an important link between hypoperfusion, embolism, and ischemic stroke. Arch Neurol. 1998;55:1475-1482.

41. Yamauchi H, Okazawa H, Kishibe Y, Sugimoto K, Takahashi M. Oxygen extraction fraction and acetazolamide reactivity in symptomatic carotid artery disease. J Neurol Neurosurg Psychiatry. 2004;75:33-37.

42. Reinhard M, Muller T, Roth M, Guschlbauer B, Timmer J, Hetzel A. Bilateral severe carotid artery stenosis or occlusion - cerebral autoregulation dynamics and collateral flow patterns. Acta Neurochir (Wien). 2003;145:1053-1060. 